Homology, Homotopy and Applications, vol.17(1), 2015, pp.281-305

\title{
SUBALGEBRAS OF THE $\mathbb{Z} / 2$-EQUIVARIANT STEENROD ALGEBRA
}

\author{
NICOLAS RICKA
}

\author{
(communicated by Daniel Dugger)
}

\begin{abstract}
The aim of this paper is to study subalgebras of the $\mathbb{Z} / 2$ equivariant Steenrod algebra (for cohomology with coefficients in the constant Mackey functor $\mathbb{F}_{2}$ ) that come from quotient Hopf algebroids of the $\mathbb{Z} / 2$-equivariant dual Steenrod algebra. In particular, we study the equivariant counterpart of profile functions, exhibit the equivariant analogues of the classical $\mathcal{A}(n)$ and $\mathcal{E}(n)$, and show that the Steenrod algebra is free as a module over these.
\end{abstract}

\section{Introduction}

It is a truism to say that the classical modulo 2 Steenrod algebra and its dual are powerful tools to study homology in particular and homotopy theory in general. In [2], Adams and Margolis have shown that sub-Hopf algebras of the modulo $p$ Steenrod algebra, or dually quotient Hopf algebras of the dual modulo $p$ Steenrod algebra have a very particular form. This allows an explicit study of the structure of the Steenrod algebra via so-called profile functions.

These facts have both a profound and meaningful consequences in stable homotopy theory and concrete, amazing applications (see [13] for many beautiful results strongly relating to the structure of the Steenrod algebra).

Using the determination of the structure of the $\mathbb{Z} / 2$-equivariant dual Steenrod algebra as an $R O(\mathbb{Z} / 2)$-graded Hopf algebroid by Hu and Kriz in [10], we show two kind of results:

1. One that parallels $[\mathbf{2}]$, characterizing families of sub-Hopf algebroids of the $\mathbb{Z} / 2$ equivariant dual Steenrod algebra, showing how to adapt classical methods to overcome the difficulties inherent to the equivariant world.

2. Answers to some natural questions arising from the essential differences between the non-equivariant and $\mathbb{Z} / 2$-equivariant stable cohomological operations with coefficients in $\mathbb{F}_{2}$ and $\underline{F}_{2}$ respectively.

Our goal is to study subobjects of the $\mathbb{Z} / 2$-equivariant Steenrod algebra via the quotients of its dual, so we start by showing how duality theory à la Boardman fits into the context of $\mathbb{Z} / 2$-equivariant stable cohomology operations in Section 3.

Received May 14, 2014, revised December 5, 2014; published on May 11, 2015. 2010 Mathematics Subject Classification: 55S10, 55S91.

Key words and phrases: cohomology operation, Hopf algebroid, equivariant Steenrod algebra. Article available at http://dx.doi.org/10.4310/HHA.2015.v17.n1.a14

Copyright (C) 2015, Nicolas Ricka. Permission to copy for private use granted. 
When considering such equivariant generalizations, one must be careful since, for an equivariant cohomology theory $E^{\star}$, freeness of stable $E^{\star}$-cohomological operations as a module over the coefficient ring $E_{\mathbb{Z} / 2}^{\star}$ (the $R O(\mathbb{Z} / 2)$-graded abelian group of $\mathbb{Z} / 2$ equivariant maps $S^{\star} \rightarrow E$ ) is not sufficient to apply Boardman's theory. The main result is Proposition 3.12, which states that the appropriate notion in $\mathbb{Z} / 2$-equivariant stable homotopy theory is freeness as an $R O(\mathbb{Z} / 2)$-graded Mackey functor for $\mathbb{Z} / 2$.

We then show how this theory applies to the $\mathbb{Z} / 2$-equivariant dual Steenrod algebra in Section 4. The essential proposition is Proposition 4.3, which asserts that the appropriate freeness condition is satisfied.

These results motivate our study of quotient Hopf algebroids of the $\mathbb{Z} / 2$-equivariant Steenrod algebra. We develop in Section 5 the required theoretical tools. After dealing with quotients of $R O(\mathbb{Z} / 2)$-graded Hopf algebroids in general, we deal with the cofreeness properties of various quotients of a given $R O(\mathbb{Z} / 2)$-graded Hopf algebroid in Theorem 6.13. The following section is devoted to the application of these ideas to the particular case of the $\mathbb{Z} / 2$-equivariant dual Steenrod algebra. We define the appropriate notion of profile function in the equivariant case in Definition 5.7 and deal with three questions:

1. Is there unicity of a pair of profile functions providing the same algebra quotient, and if yes, is there a preferred choice of profile functions?

2. Under what conditions on a pair of profile functions is the associated quotient algebra a quotient Hopf algebroid?

3. Under what additional conditions is this quotient Hopf algebroid free over $\left(H \underline{\mathbb{F}}_{\star}\right)_{\mathbb{Z} / 2}$ ?

These questions lead, respectively, to the notion of minimal pair, the well-known notion of Hopf ideal, and the notion of free pair.

We end the paper by exhibiting in Section 6 some particular sub-Hopf algebras corresponding to the non-equivariant $\mathcal{A}(n)$ and $\mathcal{E}(n)$, and exhibit some properties of these algebras. The principal result is Theorem 6.14 , which we apply to the particular cases $\mathcal{A}^{\star}(n)$ and $\mathcal{E}^{\star}(n)$ to get the following corollary.

Corollary (Corollary 6.15). For all $n \geqslant 0$, the Hopf algebroid $\left(H \underline{\mathbb{F}}_{2}, \mathcal{A}_{\star}\right)$ is cofree as a comodule over its quotients $\mathcal{E}(n)_{\star}$ and $\mathcal{A}_{\star}(n)$, and these quotient Hopf algebroids are cofree over each other.

Using duality theory for modules à la Boardman, this gives the next principal result of this paper.

Theorem 1.1. For all $n \geqslant 0$, the $\mathbb{Z} / 2$-equivariant Steenrod algebra $H \underline{\mathbb{F}}_{2}^{\star}{ }_{\mathbb{Z} / 2} H \mathbb{F}_{2}$ is free as a module over $\mathcal{E}(n)^{\star}$ and $\mathcal{A}(n)^{\star}$, and these algebras are free over each other.

As an application, we compute the $\mathbb{Z} / 2$-equivariant modulo 2 cohomology of the spectrum representing $K$-theory with reality. This type of result is essential when it comes to computing the Adams spectral sequence converging to $K$-theory with reality, as it allows us to use a change of coefficients property in the extension groups appearing in the $E^{2}$-page of the spectral sequence. This was one of the motivations of this work. We refer the interested reader to the ext charts appearing at the end of [5] for applications of the analogous non-equivariant result to the computation of some ko-cohomology groups. 


\section{Acknowledgments}

I want to thank my advisor, Geoffrey Powell, for both the very stimulating mathematical discussions we had and his careful readings of early expositions of the results presented in this paper.

\section{Preliminaries}

\subsection{Equivariant cohomology theories and $R O(\mathbb{Z} / 2)$-gradings}

Recall that the real representation ring of $\mathbb{Z} / 2, R O(\mathbb{Z} / 2)$ is isomorphic to $\mathbb{Z} \oplus \mathbb{Z} \alpha$, where 1 stands for the one-dimensional trivial representation and $\alpha$ stands for the sign representation. Consider the category of $\mathbb{Z} / 2$-equivariant spectra indexed over a complete universe $\mathbb{Z} / 2 \mathcal{S H}$. We consider its usual model category structure, so that its associated homotopy category is the $\mathbb{Z} / 2$-equivariant stable homotopy category $\mathbb{Z} / 2 \mathcal{S H}$. We refer to $[\mathbf{1 2}, \mathbf{8}]$ for the constructions and definitions. The two following properties of $\mathbb{Z} / 2 \mathcal{S} p$ are of particular importance.

Remark 2.1. 1. We implicitely chose a model for the category of $\mathbb{Z} / 2$-equivariant spectra that has good monoidal properties, such as orthogonal equivariant $\mathbb{Z} / 2$ spectra.

2. The meaning of the indexation over a complete universe is that the smash product with all the spheres $S^{V}$ obtained as the one-point compactifcation of the real representations $V$ of $\mathbb{Z} / 2$ is inverted up to homotopy.

Notation 2.2. $\quad$ - The $\star$ superscript will always denote an $R O(\mathbb{Z} / 2)$-graded object, whereas the $*$ denotes a $\mathbb{Z}$-graded one.

- There are ring morphisms

$$
i: \mathbb{Z} \hookrightarrow R O(\mathbb{Z} / 2)
$$

(subring of trivial virtual representations),

$$
\operatorname{dim}: R O(\mathbb{Z} / 2) \rightarrow \mathbb{Z},
$$

which associates to a representation $V$ its virtual dimension, and

$$
\text { twist }: R O(\mathbb{Z} / 2) \rightarrow \mathbb{Z}
$$

which is defined by twist $(k+l \alpha)=l$.

Definition 2.3. $\quad$ Let $\mathcal{O}$ be the full subcategory of $\mathbb{Z} / 2 \mathcal{S H}$ whose objects are $S^{0}$ and $\mathbb{Z} / 2_{+}$. A Mackey functor for $\mathbb{Z} / 2$ is an additive functor $\mathcal{O} \rightarrow \mathcal{A} b$.

- For $X$ and $Y$ two $\mathbb{Z} / 2$-spectra, the stable classes of maps from $X$ to $Y$, denoted $[X, Y]$, is naturally an $R O(\mathbb{Z} / 2)$-graded Mackey functor

$$
\mathbb{Z} / 2 \mathcal{S} \mathcal{H}^{o p} \times \mathbb{Z} / 2 \mathcal{S H} \rightarrow \mathcal{M}^{R O(\mathbb{Z} / 2)}
$$

defined, for $V \in R O(\mathbb{Z} / 2)$, by

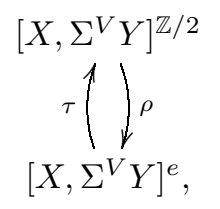


where, because of the adjunction

$$
\left[\mathbb{Z} / 2_{+} \wedge X, \Sigma^{V} Y\right]^{\mathbb{Z} / 2} \cong\left[X, \Sigma^{V} Y\right]^{e} \cong\left[X, \mathbb{Z} / 2_{+} \wedge \Sigma^{V} Y\right]^{\mathbb{Z} / 2},
$$

the restriction and transfer of these Mackey functors are induced by the projection $\mathbb{Z} / 2_{+} \rightarrow S^{0}$ and its Spanier-Whitehead dual $S^{0} \rightarrow \mathbb{Z} / 2_{+}$, respectively.

Notation 2.4. Let $M$ be a Mackey functor. If $\rho_{M}: M_{\mathbb{Z} / 2} \rightarrow M_{e}$ denotes the restriction of $M, \tau_{M}: M_{e} \rightarrow M_{\mathbb{Z} / 2}$ denotes its transfer, and $\theta_{M}: M_{e} \rightarrow M_{e}$ denotes the action of $\mathbb{Z} / 2$ on $M_{e}$, we represent $M$ by the following diagram:

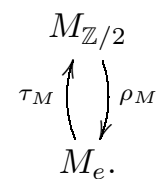

Observe that, since $\theta_{M}=\rho_{M} \tau_{M}-1$, this diagram suffices to determine $M$. We denote

$$
(-)_{e}: \mathcal{M} \rightarrow \mathbb{Z}[\mathbb{Z} / 2]-\bmod
$$

and

$$
(-)_{\mathbb{Z} / 2}: \mathcal{M} \rightarrow \mathbb{Z}-\bmod
$$

the evaluation functors associated to the two objects of the orbit category for $\mathbb{Z} / 2$.

In particular, $\mathbb{Z} / 2$-equivariant cohomology theories are functors $\mathbb{Z} / 2 \mathcal{S H}^{o p} \rightarrow$ $\mathcal{M}^{R O(\mathbb{Z} / 2)}$ satisfying equivariant analogues of the Eilenberg-Steenrod axioms.

We now turn to some elementary definitions in the abelian category of $R O(\mathbb{Z} / 2)$ graded objects.

Notation 2.5. Let $\left(\mathcal{B}, \otimes, 1_{\mathcal{B}}\right)$ be a closed symmetric monoidal abelian category. Denote $\mathcal{B}^{R O(\mathbb{Z} / 2)}$ the category of $R O(\mathbb{Z} / 2)$-graded objects. For an element $x$ of an $R O(\mathbb{Z} / 2)$ graded object $M^{\star}$, denote $|x| \in R O(\mathbb{Z} / 2)$ its degree.

As the underlying abelian group of $R O(\mathbb{Z} / 2)$ is in particular an abelian monoid, one can define a symmetric monoidal structure on $\mathcal{B}^{R O(\mathbb{Z} / 2)}$ in a very natural way.

Definition 2.6. Let $\otimes: \mathcal{B}^{R O(\mathbb{Z} / 2)} \times \mathcal{B}^{R O(\mathbb{Z} / 2)} \rightarrow \mathcal{B}^{R O(\mathbb{Z} / 2)}$ denote the functor

$$
(x \otimes y)_{\star}=\bigoplus_{V \oplus W=\star} x_{V} \otimes y_{W} .
$$

For two $R O(\mathbb{Z} / 2)$-graded objects $M, N, \tau: M \otimes N \rightarrow N \otimes M$ is the natural isomorphism determined by $\left(\tau: M^{V} \otimes N^{W} \rightarrow N^{W} \otimes M^{V}\right)=(-1)^{\operatorname{dim}(V) \operatorname{dim}(W)} \tau_{\mathcal{B}}$, where $\tau_{\mathcal{B}}$ denotes the twist isomorphism in the category $\mathcal{B}$.

Lemma 2.1. The triple $\left(\otimes, \tau, 1_{\mathcal{B}}\right)$, with the obvious coherence morphisms, give $\mathcal{B}^{R O(\mathbb{Z} / 2)}$ the structure of a closed symmetric monoidal category, where Hom is given in degree $\star$ by morphisms that increase the degree by $\star$.

Proof. The proof, which is formally the same as in the classical case, is left to the reader.

Notation 2.7. 1. A (commutative, unitary) $R O(\mathbb{Z} / 2)$-graded ring in $\mathcal{B}$ is a (commutative, unitary) monoid in the category $\left(\otimes, \tau, 1_{\mathcal{B}}\right)$. 
2. For a commutative ring $H, H-M o d$ (the category of $H$-modules) and $H-$ Bimod (the category of $H$-bimodules) are defined via the usual diagrams.

3. For $R$ a cocommutative comonoid in $\left(\otimes, \tau, 1_{\mathcal{B}}\right)$, the category of $R$-comodules is defined as usual.

2.2. The $\mathbb{Z} / 2$-spectrum $H \underline{\mathbb{F}}_{2}$

Definition 2.8. Denote $\underline{\pi}_{\star}: \overline{\mathbb{Z} / 2} \mathcal{S H} \rightarrow \mathcal{M}^{R O(\mathbb{Z} / 2)}$ the functor $X \mapsto\left[S^{\star}, X\right]$.

Using the functor $\underline{\pi}_{*}$, given by restricting $\pi_{\star}$ to integer gradings, one can extend the definition of Postnikov tower to the $\mathbb{Z} / 2$-equivariant setting. This provides the appropriate notion of ordinary cohomology theory.

Proposition 2.9. The $\mathbb{Z} / 2$-equivariant Postnikov tower defines a $t$-structure on the $\mathbb{Z} / 2$-equivariant stable homotopy category whose heart is isomorphic to the category $\mathcal{M}$ of Mackey functors for the group $\mathbb{Z} / 2$. In particular, one has a functor

$$
H: \mathcal{M} \rightarrow \mathbb{Z} / 2 \mathcal{S H}
$$

which sends short exact sequences of Mackey functors to distinguished triangles of $\mathbb{Z} / 2$-equivariant spectra.

Proof. This proposition summarizes the results of [12, Proposition I.7.14] and [11, Theorem 1.13] in the particular case of the group with two elements.

Definition 2.10. Denote by $\underline{\mathbb{Z}}$ the Mackey functor

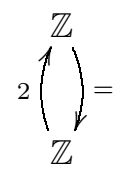

and denote by $\mathbb{F}_{2}$ the Mackey functor

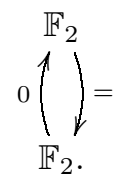

Remark 2.11. The constant Mackey functors in general, and $\mathbb{F}_{2}, \underline{\mathbb{Z}}$ in particular, play a special role in this context. One reason is that equivariant Eilenberg-MacLane spectra with coefficients in constant Mackey functors are exactly the 0th-slices in the sense of the slice filtration (see [9, Proposition 4.47]). The spectrum $H \underline{Z}$ is even more particular since it identifies with $P_{0}^{0}\left(S^{0}\right)$ by [9, Corollary 4.51]. This particular role of $H \underline{Z}$ was first observed by Dugger in [7], studying an Atiyah-Hirzebruch spectral sequence for $K \mathbb{R}$-theory, which was really the slice spectral sequence of $K \mathbb{R}$.

\subsection{The coefficient ring for $\mathrm{HF}_{2}$}

We now write down the structure of the coefficient ring for $\mathrm{HF}_{2}$-cohomology. For the complete computation, see [10, p.371].

Recall from Remark 2.1 that for a real representation, $V$ of $\mathbb{Z} / 2$, the object $S^{V}$ stands for the one-point compactification of $V$ as a $\mathbb{Z} / 2$-space. 
Proposition 2.12. The $R O(\mathbb{Z} / 2)$-graded Mackey functor $H \underline{\mathbb{F}}_{\alpha_{\star}}$ is represented in the following picture.

The symbol • stands for the Mackey functor

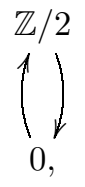

and $L$ stands for

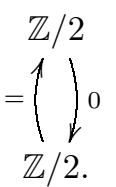

Finally, L_ represents the Mackey functor that consists only of $\mathbb{F}_{2}$ concentrated in $(-)_{e}$. A vertical line represents the product with the Euler class a, which is the class of the map $S^{0} \hookrightarrow S^{\alpha}$. This product induces one of the following Mackey functor maps:

- the identity of $\bullet$,

- the unique non-trivial morphism $L \rightarrow \bullet$,

- the unique non-trivial morphism $\bullet \hookrightarrow \mathbb{F}_{2}$.

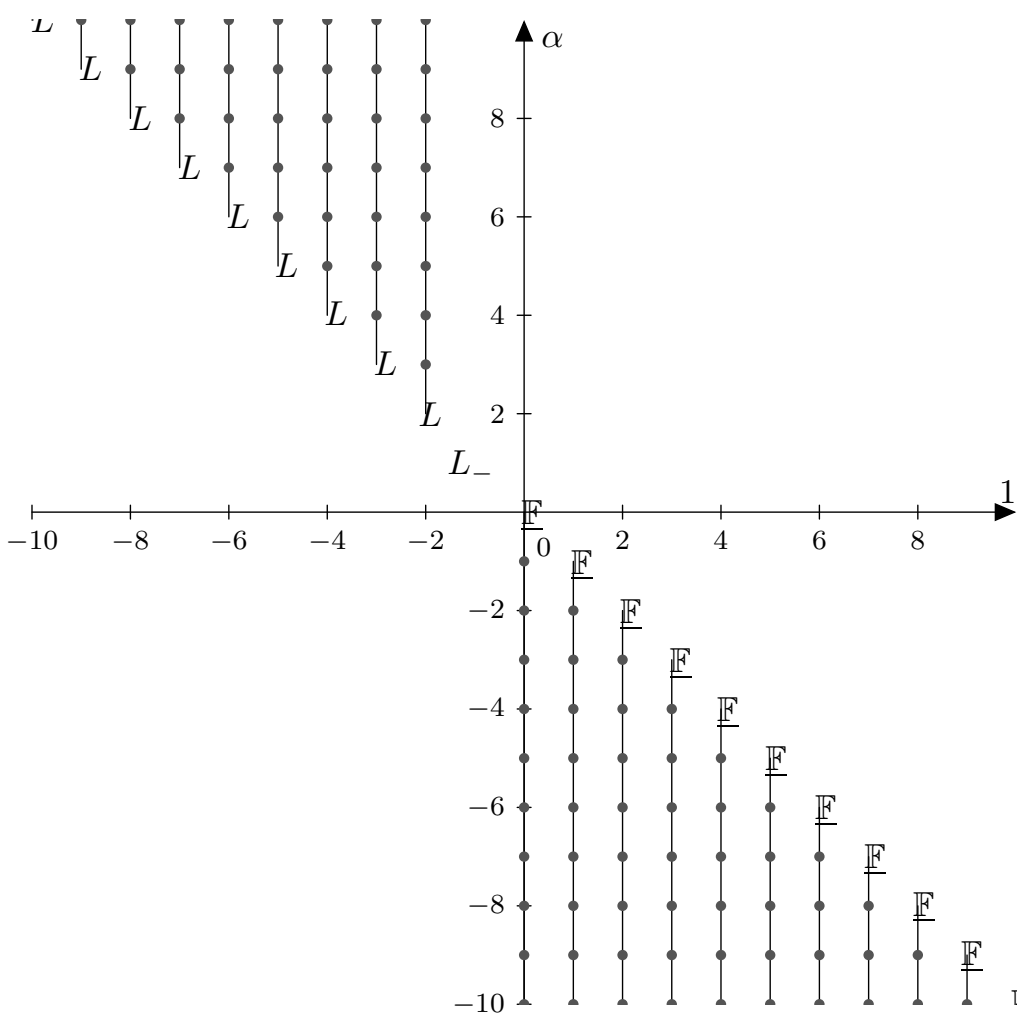


Notation 2.13. We call $\sigma^{-1}$ the non-trivial element in degree $(1-\alpha)$, so that $\left(H \mathbb{F}_{2}\right)_{\mathbb{Z} / 2}$ contains $\mathbb{F}_{2}\left[a, \sigma^{-1}\right]$ as a subalgebra.

We finish this subsection with a lemma about Mackey functors, relating the $\mathbb{Z}[a] /$ $(2 a)$-module structure on $\left([-,-]^{\star}\right)_{\mathbb{Z} / 2}$ with the $R O(\mathbb{Z} / 2)$-graded Mackey functor structure of $[-,-]^{\star}$.

Lemma 2.2. Let $E$ be a $\mathbb{Z} / 2$-spectrum.

1. $\operatorname{Im}(a)=\operatorname{Ker}(\rho)$, where $\rho:\left(E_{\star}\right)_{\mathbb{Z} / 2} \rightarrow\left(E_{\star}\right)_{e}$ stands for the restriction of the Mackey functor $E_{\star}$.

2. $\operatorname{Ker}(a)=\operatorname{Im}(\tau)$, where $\tau$ is the transfer.

Proof. These are consequences of the existence of the long exact sequence associated to the distinguished triangle

$$
\mathbb{Z} / 2_{+} \rightarrow S^{0} \rightarrow S^{\alpha}
$$

in the stable $\mathbb{Z} / 2$-equivariant category.

1. Apply the exact functor $\left[-, \Sigma^{-\star} E\right]^{\mathbb{Z} / 2}$ to the triangle. We have

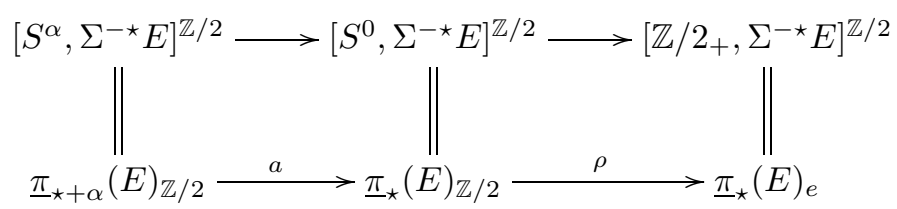

where the rows are exact. The first point follows.

2. Apply the exact functor $\left[S^{\star},(-) \wedge E\right]_{e}$ to the triangle. We have

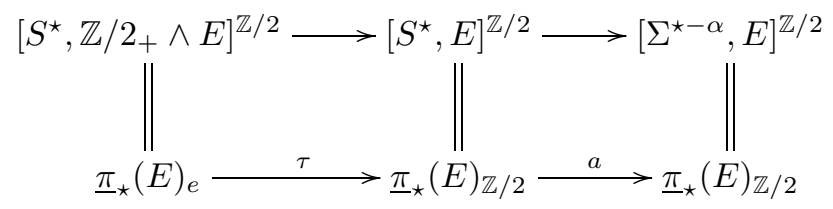

where the rows are exact. The second point follows.

\section{Operations in $R O(\mathbb{Z} / 2)$-graded cohomology}

\section{1. $R O(\mathbb{Z} / 2)$-graded Hopf algebroids}

The role of this section is to set up the appropriate structure we need to deal with homology cooperations. These constructions are the $R O(\mathbb{Z} / 2)$-graded analogue of the standard notions of Hopf algebroid and the machinery considered by Boardman [4]. We now define two monoidal structures on the category of $H$-bimodules.

Definition 3.1. Let $H$ be a commutative $R O(\mathbb{Z} / 2)$-graded ring.

1. Let $\underset{(r, l)}{\otimes}: H-\operatorname{Bimod} \times H-\operatorname{Bimod} \rightarrow H-\operatorname{Bimod}$ be the tensor product over $H$, with respect to the right $H$-module structure on the first argument and the left $H$-module structure on the second. The unit for this structure is $H$ with the $H$-bimodule structure induced by the product on both sides. 
2. Let $\underset{(l, l)}{\otimes}: H-\operatorname{Bimod} \times H-\operatorname{Bimod} \rightarrow H-\operatorname{Bimod}$ be the tensor product over $H$, with respect to the left $H$-module structure on both arguments. The $H$ bimodule structure on $M \underset{(l, l)}{\otimes} N$ is given by $h(m \otimes n) h^{\prime}:=(m h) \otimes\left(n h^{\prime}\right)$ for $h, h^{\prime} \in H$ and $n \in N, M \in M$. Again, $H$ is the unit for this monoidal structure.

3. When $A$ is an $H$-bimodule and $M$ is a right $H$-module (resp. a left $H$-module), we can still define $M \underset{(r, l)}{\otimes} A$ (resp. $M \underset{(l, l)}{\otimes} A, A \underset{(r, l)}{\otimes} M$ ) as a right (resp. right, left) $H$-module.

Notation 3.2. Let $H$ be a commutative $R O(\mathbb{Z} / 2)$-graded ring.

1. $H-A l g$ is the category of monoids in $(H-\bmod , \otimes, H)$ and monoid morphisms.

2. $A l g$ is the category of $R O(\mathbb{Z} / 2)$-graded algebra, that is $\mathbb{Z}-A l g$ where $\mathbb{Z}$ is concentrated in degree $0 \in R O(\mathbb{Z} / 2)$.

3. An $R O(\mathbb{Z} / 2)$-graded Hopf algebroid $(H, A)$ is a cogroupoid object in $\mathcal{A} b^{R O(\mathbb{Z} / 2)}$.

Equivalently, an $R O(\mathbb{Z} / 2)$-graded Hopf algebroid structure consists in the following data.

Definition 3.3 ( $c f$ [15, Definition A1.1.1]). Let $(H, A)$ be a pair of $R O(\mathbb{Z} / 2)$-graded algebras. Denote by $\mu$ the product of the algebra $A$. An $R O(\mathbb{Z} / 2)$-graded Hopf algebroid structure on $(H, A)$ consists of

1. a left unit $\eta_{L}: H \rightarrow A$,

2. a right unit $\eta_{R}: H \rightarrow A$,

3. a coproduct $\Delta: A \rightarrow A \underset{(r, l)}{\otimes} A$,

4. a counit $\epsilon: A \rightarrow H$, and

5. an antipode $c: A \rightarrow A$,

satisfying the following requirements:

1. $A$ has a $H$-bimodule structure, induced by $\eta_{L}$ (for the left module structure) and $\eta_{R}$ (for the right module structure), and $\Delta$ and $\epsilon$ are $H$-bimodule morphisms.

2. The equalities

- $\epsilon \eta_{L}=\epsilon \eta_{R}=I d_{H}$

- $\left(I d_{A} \underset{(r, l)}{\otimes} \epsilon\right) \circ \Delta=\left(\epsilon \underset{(r, l)}{\otimes} I d_{A}\right) \circ \Delta=I d_{A}$

- $\left(I d_{A} \underset{(r, l)}{\otimes} \Delta\right) \circ \Delta=\left(\Delta \underset{(r, l)}{\otimes} I d_{A}\right) \circ \Delta$

- $c \eta_{L} \stackrel{(r, l)}{=} \eta_{R}$

- $c \eta_{R}=\eta_{L}$

- $c \circ c=I d_{A}$

are satisfied. 
3. There exist dotted arrows making the following diagram commute:

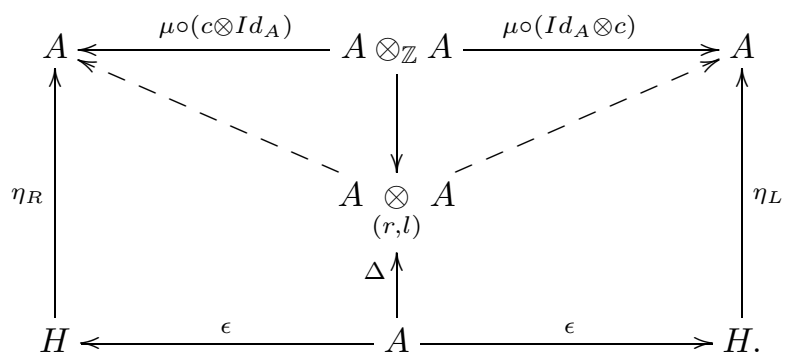

References for the proof: See [15, definition A1.1.1] and subsequent discussion (p. 302 ) for this property in the category of $\mathbb{N}$-graded algebras over a ring $K$. The proof is, mutatis mutandis, the same in the $R O(\mathbb{Z} / 2)$-graded context.

We have all the usual notions attached to Hopf algebroids in the $R O(\mathbb{Z} / 2)$-graded context, and, in particular, the following:

Definition 3.4. Let $(H, A)$ be an $R O(\mathbb{Z} / 2)$-graded Hopf algebroid; we say that $x \in A$ is primitive if $\Delta(x)=1 \otimes_{H} x+x \otimes_{H} 1$.

\subsection{Duality between modules and comodules à la Boardman}

Following Boardman [4], we now study a form of duality between modules and comodules that mimics the duality between cohomological operations and homological cooperations in the non-equivariant context.

Definition 3.5 ([4, definitions in $\S 10$ and Definition 11.11]).

1. Let $A^{\star}$ be an $H$-bimodule and an $R O(\mathbb{Z} / 2)$-graded ring. An $A^{\star}$-module à la Boardman is an $R O(\mathbb{Z} / 2)$-graded filtered complete Hausdorff $H$-module $M$, together with a continuous $H$-module morphism

$$
\lambda: A^{\star} \underset{(r, l)}{\otimes} M \rightarrow M
$$

with the usual commutative diagrams defining a module over a ring.

2. Let $A_{\star}$ be an $R O(\mathbb{Z} / 2)$-graded Hopf algebroid. An $A_{\star}$-comodule à la Boardman is an $R O(\mathbb{Z} / 2)$-graded filtered complete Hausdorff $H$-module $M$, together with a continuous $H$-module morphism

$$
\rho: M \rightarrow M \underset{(l, l)}{\hat{\otimes}} A_{\star},
$$

where the action of $H$ on $M \hat{\otimes} A_{\star}$ is given by $h(m \otimes s)=m \otimes \eta_{R}(h) s$, for $m \otimes$ $s \in M \hat{\otimes} A_{\star}$ and $h \in H$.

$(l, l)$

Remark 3.6. The difference between these notions and the more naive notions of module and comodule (see Definition 2.7) is the completion of the tensor product $\left(M \hat{\otimes} A_{\star}\right)$. 
The importance of these notions for us appears in the following extension to the $R O(\mathbb{Z} / 2)$-graded context of a result of Boardman $[\mathbf{4}]$.

Proposition 3.7 ([4, theorem 11.13]). Let $A_{\star}$ be an $R O(\mathbb{Z} / 2)$-graded Hopf algebroid, and suppose that $A_{\star}$ is a free left $H$-module. Denote by $A^{\star}=\operatorname{Hom}_{H-M o d}\left(A_{\star}, H\right)$. Then there is an equivalence of categories between the category of $A^{\star}$-modules and the category of $A_{\star}$-comodules.

\subsection{Hopf algebroid structure on cooperations in homology with respect to a flat commutative ring spectrum}

Definition 3.8. Let $E$ be a commutative $\operatorname{ring} \mathbb{Z} / 2$-spectrum. We say that $E$ is flat if the $E_{\star}^{\mathbb{Z} / 2}$-module $E_{\star}^{\mathbb{Z} / 2} E$ is flat.

Proposition 3.9. Let $E$ be a flat commutative ring $\mathbb{Z} / 2$-spectrum. Then the unit $\eta$ : $S^{0} \rightarrow E$ and multiplication $\mu: E \wedge E \rightarrow E$ induce an $R O(\mathbb{Z} / 2)$-graded Hopf algebroid structure on the pair $\left(E_{\star}^{\mathbb{Z} / 2}, E_{\star}^{\mathbb{Z} / 2} E\right)$. Moreover, this Hopf algebroid is flat.

For any $\mathbb{Z} / 2$-spectrum $X, E_{\mathbb{Z} / 2}^{\star}(X)$ with the topology induced by the skeletal filtration is a comodule à la Boardman over this $R O(\mathbb{Z} / 2)$-graded Hopf algebroid.

Proof. The constructions of [1, Chapter 12 and 13], [15, Section 2.2] extend to the $R O(\mathbb{Z} / 2)$-graded setting. The comodule structure is given by an $R O(\mathbb{Z} / 2)$-graded analogue of $[\mathbf{4}$, Theorem 4.2].

Thus, Boardman's Theorem 3.5 holds in this setting, and thus provides an equivalence between the category of $E_{\star}^{\mathbb{Z} / 2} E$-comodules à la Boardman and the category of $H_{o m}{ }_{E_{\star}^{\mathbb{Z} / 2}}\left(E_{\star}^{\mathbb{Z} / 2} E, E_{\star}^{\mathbb{Z} / 2}\right)$-modules. We want to compare this $\operatorname{Hom}_{E_{\star}^{\mathbb{Z} / 2}}\left(E_{\star}^{\mathbb{Z} / 2} E, E_{\star}^{\mathbb{Z} / 2}\right)-$ module structure to the more familiar $E_{\mathbb{Z} / 2}^{\star} E$-module structure on $E^{\star}$-cohomology.

In general, the relationship between stable cohomological operations, given by $[E, E]_{\mathbb{Z} / 2}^{\star}$, and cooperations, given by $\left(E_{\star}^{\mathbb{Z} / 2}, E_{\star}^{\mathbb{Z} / 2} E\right)$, is delicate. In order to obtain an isomorphism

$$
\operatorname{Hom}_{E_{\star}^{\mathbb{Z} / 2}}\left(E_{\star}^{\mathbb{Z} / 2} E, E_{\star}^{\mathbb{Z} / 2}\right) \cong E_{\mathbb{Z} / 2}^{\star} E
$$

we need the following additional hypothesis on $E$ :

Hypothesis 3.10. The $R O(\mathbb{Z} / 2)$-graded Mackey functor $E_{\star} E$ is isomorphic to a direct sum $\bigoplus_{i \in I} \Sigma^{V_{i}} E_{\star}$ as an $E_{\star}$-module for some $V_{i} \in R O(\mathbb{Z} / 2)$.

Generally, for a commutative ring $\mathbb{Z} / 2$-spectrum $E$, freeness of an $E$-module can be understood in purely algebraic terms.

Proposition 3.11. Let $X$ be an E-module such that $\underline{\pi}_{\star} X$ is isomorphic (as an $R O(\mathbb{Z} / 2)$-graded Mackey functor) to $\bigoplus_{i \in I} \Sigma^{V_{i}} E_{\star}$, for some $V_{i} \in R O(\mathbb{Z} / 2)$. Then, there exists an $E$-module weak equivalence $\bigvee_{i \in I} \Sigma^{V_{i}} E \simeq X$. 
Proof. Let $\left\{b_{i}\right\}_{i \in I}$ be a basis of $E_{\star} X$ as an $E_{\star}$-module, with $\left|b_{i}\right|=V_{i}$. Consider

$$
\bigvee_{i \in I} S^{V_{i}} \stackrel{\vee b_{i}}{\rightarrow} X
$$

This provides an $E$-module morphism

$$
\bigvee_{i \in I} \Sigma^{\operatorname{deg}\left(b_{i}\right)} E \rightarrow X
$$

This $\mathbb{Z} / 2$-spectrum map induces an isomorphism in homotopy Mackey functors, and thus is a weak equivalence.

Proposition 3.12. Suppose that E satisfies Hypothesis 3.10. Then there is an $R O(\mathbb{Z} / 2)$-graded ring and $E_{\star}^{\mathbb{Z} / 2}$-module isomorphism

$$
\operatorname{Hom}_{E_{\star}^{\mathbb{Z} / 2}}\left(E_{\star}^{\mathbb{Z} / 2} E, E_{\star}^{\mathbb{Z} / 2}\right) \cong E_{\mathbb{Z} / 2}^{\star} E,
$$

where the ring structures are

1. induced by composition of morphisms $E \rightarrow E$ for $E_{\mathbb{Z} / 2}^{\star} E=\pi_{\star}(E n d(E))_{\mathbb{Z} / 2}$, and

2. given by the composite $\operatorname{Hom}_{E_{\star}^{\mathbb{Z} / 2}}\left(E_{\star}^{\mathbb{Z} / 2} E, E_{\star}^{\mathbb{Z} / 2}\right) \otimes_{E_{\star}^{\mathbb{Z} / 2}} \operatorname{Hom}_{E_{\star}^{\mathbb{Z} / 2}}\left(E_{\star}^{\mathbb{Z} / 2} E, E_{\star}^{\mathbb{Z} / 2}\right) \rightarrow$ $\operatorname{Hom}_{E_{\star}^{\mathbb{Z} / 2}}\left(E_{\star}^{\mathbb{Z} / 2} E \otimes_{E_{\star}^{\mathbb{Z} / 2}}\left(E_{\star}^{\mathbb{Z} / 2} E, E_{\star}^{\mathbb{Z} / 2}\right) \stackrel{\Delta^{*}}{\rightarrow} \operatorname{Hom}_{E_{\star}^{\mathbb{Z} / 2}}\left(E_{\star}^{\mathbb{Z} / 2} E, E_{\star}^{\mathbb{Z} / 2}\right)\right.$.

Proof (indications, see [4, proof of Theorem 9.25]). As $E$ satisfies Hypothesis 3.10, choose a basis $\left\{b_{i}\right\}_{i \in I}$ of the $E_{\star}^{\mathbb{Z} / 2}$-module $E_{\star}^{\mathbb{Z} / 2} E$, so that there is a weak equivalence $E \wedge E \simeq \bigvee_{i} \Sigma^{\operatorname{deg}\left(b_{i}\right)} E$. Then the map

$$
\begin{aligned}
\pi^{-1}: \operatorname{Hom}_{E_{\star}^{\mathbb{Z} / 2}}\left(E_{\star}^{\mathbb{Z} / 2} E, E_{\star}^{\mathbb{Z} / 2}\right) & \rightarrow \pi_{\star} \operatorname{Hom}_{E-M o d}(E \wedge E, E) \\
& \cong \pi_{\star}(\operatorname{Hom}(E, E))=E_{\mathbb{Z} / 2}^{\star} E
\end{aligned}
$$

defined for $f: b_{i} \mapsto e_{i} \in E_{\star}$ by the formula

$$
\pi^{-1}(f): \bigvee_{i} \Sigma^{\operatorname{deg}\left(b_{i}\right)} E \stackrel{\vee e_{i} \wedge E}{\longrightarrow} E \wedge E \stackrel{\mu}{\longrightarrow} E
$$

provides an inverse isomorphism to the map

$$
\pi_{\star}: E_{\mathbb{Z} / 2}^{\star} E \cong \pi_{\star} \operatorname{Hom}_{E-M o d}(E \wedge E, E) \rightarrow \operatorname{Hom}_{E_{\star}^{Z} / 2}\left(E_{\star}^{\mathbb{Z} / 2} E, E_{\star}^{\mathbb{Z} / 2}\right) .
$$

Commutativity of the diagram [4, Diagram 4.18] implies that this isomorphism is a ring isomorphism.

Corollary 3.13 (of 3.12). Let $E$ be a ring $\mathbb{Z} / 2$-spectrum satisfying Hypothesis 3.10. Then, by the isomorphism provided by Proposition 3.12, a stable $E^{\star}$-cohomology operation $x \in E_{\mathbb{Z} / 2}^{\star} E$ is indecomposable if and only if $x^{\vee} \in E_{\star}^{\mathbb{Z} / 2} E$ is primitive.

\section{The $\mathbb{Z} / 2$-equivariant Steenrod algebra and duality}

We now turn to the study of the central object of this paper: the $\mathbb{Z} / 2$-equivariant Steenrod algebra. 
Definition 4.1. Let $\mathcal{A}^{\star}=H \underline{\mathbb{F}}_{2}^{\star}{ }_{\mathbb{Z} / 2}^{\star} H \underline{\mathbb{F}}_{2}$ be the algebra of stable $H \underline{\mathbb{F}}_{2}$-cohomology operations.

Recall that $\mathrm{Hu}$ and $\mathrm{Kriz}[\mathbf{1 0}]$ computed a presentation of the $\mathbb{Z} / 2$-equivariant modulo 2 dual Steenrod algebra

$$
\mathcal{A}_{\star}:=H \underline{\mathbb{F}}_{\underline{\mathbb{N}}_{\star}}{ }^{\mathbb{Z} / 2} H \underline{\mathbb{F}_{2}}=H \underline{\mathbb{F}}_{\star}{ }_{\star}^{\mathbb{Z} / 2}\left[\xi_{i+1}, \tau_{i} \mid i \geqslant 0\right] / I,
$$

where $I$ is the ideal generated by the relation $\tau_{i}^{2}=a \xi_{i+1}+\left(a \tau_{0}+\sigma^{-1}\right) \tau_{i+1}$, and $\sigma^{-1}$ is the class defined in Notation 2.13. From this, we deduce the following result.

Proposition 4.2. The $H \underline{\mathbb{F}}_{\star}{ }_{\star}^{\mathbb{Z} / 2}$-module $H \underline{\mathbb{F}}_{\star}{ }_{\star}^{\mathbb{Z} / 2} H \underline{\mathbb{F}_{2}}$ is free over

$$
\mathcal{B}_{m}:=\left\{\Pi_{i, j} \tau_{i}^{\epsilon_{i}} \xi_{j}^{n(j)}, n(j) \in \mathbb{N}, \epsilon(i) \in\{0,1\}\right\} .
$$

We call $\mathcal{B}_{m}$ the monomial basis of $\mathrm{HE}_{\underline{\mathbb{F}}_{\star}}{ }_{\star}^{\mathbb{Z} / 2} H \underline{\mathbb{F}}_{2}$.

Proof. The proof is quite straightforward, but we include it here for completeness.

We show that the $\mathrm{HF}_{2}{ }_{\star}$-module morphism

$$
\phi: H \underline{\mathbb{F}}_{2}\{\mathcal{B M}\} \rightarrow \mathcal{A}_{\star}
$$

is an isomorphism.

Let $R$ be the ideal generated by $a \tau_{k+1}+\eta_{R}\left(\sigma^{-1}\right) \xi_{k+1}-\tau_{k}^{2}$ for $k \geqslant 0$ so that $\mathcal{A}_{\star} \cong$ $H \underline{\mathbb{F}}_{{ }_{\star}}\left[\xi_{i+1}, \tau_{i} \mid i \geqslant 0\right] / R$.

- Surjectivity: Surjectivity follows from the definition of $\mathcal{B M}$. Let

$$
\xi_{1}^{i_{1}} \ldots \xi_{n}^{i_{n}} \tau_{0}^{j_{1}} \ldots \tau_{m}^{j_{m}}
$$

be an element of $\mathcal{B M}$. For all $k$ such that $j_{k} \geqslant 2$, write

$$
\xi_{1}^{i_{1}} \ldots \xi_{n}^{i_{n}} \tau_{0}^{j_{1}} \ldots \tau_{m}^{j_{m}} \equiv \xi_{1}^{i_{1}} \ldots \xi_{n}^{i_{n}}\left(\Pi_{k \mid j_{k} \leqslant 1} \tau_{j_{k}}\right)\left(\Pi_{k \mid j_{k} \geqslant 2} \tau_{k}^{j_{k}-2}\left(a \tau_{k+1}+\eta_{R}\left(\sigma^{-1}\right) \xi_{k+1}\right)\right.
$$

modulo $R$. By induction over $\max \left\{j_{k}\right\}$, there is an element of $H \underline{\mathbb{F}}_{2_{\star}}\{\mathcal{B M}\}$ whose image by $\phi$ is $\xi_{1}^{i_{1}} \ldots \xi_{n}^{i_{n}} \tau_{0}^{j_{1}} \ldots \tau_{m}^{j_{m}}$.

- Injectivity: This is shown analogously to the non-equivariant odd case. First, see that $\operatorname{Ker}(\phi) \cong H \mathbb{F}_{2_{\star}}\{\mathcal{B M}\} \cap R$. But for all $0 \neq r \in R, \exists i_{1}, \ldots, i_{n}, j_{1}, \ldots, j_{k}$ and $\exists k \geqslant k_{0} \geqslant 0$ such that $j_{k} \geqslant 2$ and $p r_{H \underline{\mathbb{F}_{2}}} \xi_{1}^{i_{1}} \ldots \xi_{n}^{i_{n}} \tau_{0}^{j_{1}} \ldots \tau_{m}^{j_{m}}(r) \neq 0$. By definition of $\mathcal{B M}, H \underline{\mathbb{F}}_{2}\{\mathcal{B M}\} \cap R=0$.

In order to apply Boardman's theorem for $\mathcal{A}_{\star}$-comodules à la Boardman, we need the following result.

Proposition 4.3. There is an isomorphism of Mackey functors

$$
H \underline{\mathbb{F}}_{\underline{F}_{\star}}\left(H \underline{\mathbb{F}_{2}}\right) \cong \bigoplus_{b \in \mathcal{B}_{m}} \Sigma^{|b|} H \underline{\mathbb{F}}_{\star} \star
$$

Proof. We first show the result in degrees indexed over trivial virtual representations $\star=* \in \mathbb{Z} \subset R O(\mathbb{Z} / 2)$. Let $F=\bigoplus_{b \in \mathcal{B}_{m}} \Sigma^{|b|} H{\underline{\mathbb{F}_{2}}}_{\star}{ }_{\star} / 2$. We construct an explicit Mackey 
functor isomorphism:

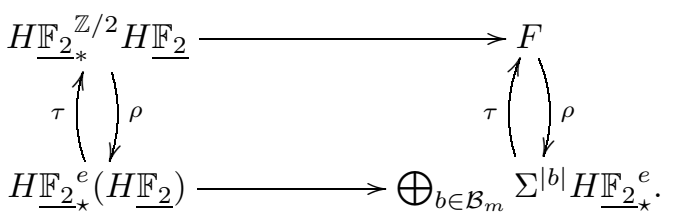

Proposition 4.2 gives precisely the isomorphism $H \underline{\mathbb{F}}_{2}^{\mathbb{Z} / 2} H \underline{F}_{2} \rightarrow F$ by restricting to integers degrees.

By definition of Eilenberg-MacLane spectra the underlying non-equivariant spectrum of $H \underline{\mathbb{F}}_{2}$ is $H \mathbb{F}_{2}$, so there is an isomorphism of $\mathbb{Z}$-graded abelian groups:

$$
H \underline{\mathbb{F}}_{*}^{e}\left(H \underline{\mathbb{F}_{2}}\right)=\underline{\pi}_{*}\left(H \underline{\mathbb{F}_{2}} \wedge H \underline{\mathbb{F}}_{2}\right)_{e}=\mathcal{A}_{*} .
$$

Recall that for all $\mathbb{Z} / 2$-spectrum $E$ whose underlying non-equivariant spectrum is $E^{u}$, $\underline{\pi}_{\star}(E)_{\mathbb{Z} / 2} \cong \pi_{\operatorname{dim}(\star)}\left(E^{u}\right) \cong \pi_{*}\left(E^{u}\right)\left[\sigma^{ \pm 1}\right]$. Thus, we obtain an $R O(\mathbb{Z} / 2)$-graded abelian groups isomorphism:

$$
\left(H \underline{\mathbb{F}}_{{ }_{*}}^{e}\left(H \underline{\mathbb{F}}_{2}\right)\right)=\underline{\pi}_{*}\left(H \underline{\mathbb{F}}_{2} \wedge H \underline{\mathbb{F}}_{2}\right)_{e}\left[\sigma^{-1}\right]=\mathcal{A}_{*}\left[\sigma^{-1}\right] .
$$

The result [10, Theorem 6.41] implies that, in integer grading, the product with the Euler class $a$ on $H \underline{\mathbb{F}}_{2} H \underline{\mathbb{F}}_{2}$ is injective. By Lemma 2.2, we know that the transfer is trivial in these degrees. Thus, the trace is trivial too, and the $\mathbb{Z} / 2$-action on $\left(H \underline{\mathbb{F}}_{\star}{ }_{\star}^{e}\left(H \underline{\mathbb{F}}_{2}\right)\right)$ is trivial.

But we already have $H \underline{\mathbb{F}}_{\star}{ }_{\star}{ }_{\star}=\mathbb{F}_{2}\left[\sigma^{ \pm 1}\right]$, so

$$
\bigoplus_{b \in \mathcal{B}_{m}} \Sigma^{|b|} H \underline{\mathbb{F}}_{\star}{ }_{\star}=\bigoplus_{b \in \mathcal{B}_{m}} \Sigma^{\operatorname{deg}(b)} \mathbb{F}_{2}
$$

with trivial $\mathbb{Z} / 2$ action.

We deduce that the $\mathbb{Z}$-graded algebra morphism

$$
\psi:\left(H \underline{\mathbb{F}}_{2}\left(H \underline{\mathbb{F}}_{2}\right)\right)_{\mathbb{Z} / 2} \rightarrow \bigoplus_{b \in \mathcal{B}_{m}} \Sigma^{|b|}\left(H \underline{\mathbb{F}}_{*}\right)_{\mathbb{Z} / 2},
$$

which sends, for all $i \geqslant 0$, the element $\sigma^{-2^{i}+1} \tau_{i} \in\left(H \underline{\mathbb{F}}_{2_{2}{ }^{i+1}-1} H \underline{\mathbb{F}}_{2}\right)_{\mathbb{Z} / 2}$ of the Steenrod algebra to $\xi_{i+1} \in \mathcal{A}^{*}=\left(H \underline{\mathbb{F}}_{2}\left(H \underline{\mathbb{F}}_{2}\right)\right)_{\mathbb{Z} / 2}$, is a $\mathbb{F}_{2}[\mathbb{Z} / 2]$-module isomorphism.

Commutation with transfer is satisfied since these morphisms are trivial.

By Lemma 2.2, we know the coimage of the restriction morphism for $H \underline{\mathbb{F}}_{2}{ }_{\star}\left(H \underline{\mathbb{F}_{2}}\right)$ and $\bigoplus_{b \in \mathcal{B}_{m}} \Sigma^{|b|} H \underline{\mathbb{F}}_{2_{\star}}$. For dimension reasons (the vector spaces are of finite dimension in each degree), these two restriction morphisms are surjective. Thus, replacing $\psi^{-1}$ by the composition of $\psi^{-1}$ with an $\mathbb{F}_{2}$-vector space isomorphism if necessary, the morphism

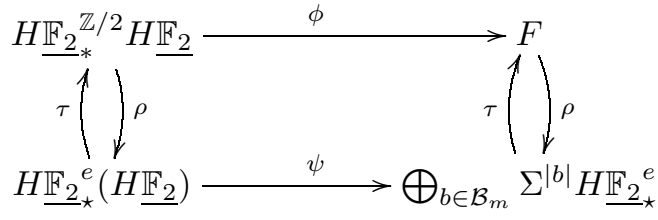


is a Mackey functor isomorphism

We finish this subsection with a comparison between the $\mathbb{Z} / 2$-equivariant dual Steenrod algebra and its non-equivariant counterpart $\mathcal{A}_{*}$, which uses the Mackey functor structure we have just determined.

Proposition 4.4. Via the identification $H \underline{\mathbb{F}}_{2}{ }_{\star}\left(H \mathbb{F}_{2}\right) \cong \mathcal{A}_{*}\left[\sigma^{ \pm 1}\right]$, the restriction of the Mackey functor $H \underline{\mathbb{F}}_{2_{\star}}\left(H \underline{\mathbb{F}}_{2}\right)$ yields an algebra map

$$
\rho: \mathcal{A}_{\star} \rightarrow \mathcal{A}_{*}\left[\sigma^{ \pm 1}\right] .
$$

Moreover, it induces a $\mathbb{Z}$-graded Hopf algebroid morphism

$$
r:\left(\left(H \underline{\mathbb{F}}_{\star}\right)_{e}, \mathcal{A}_{\star}\right) \rightarrow\left(\mathbb{F}_{2}, \mathcal{A}_{*}\right)
$$

with the $\mathbb{Z}$-graduation induced by $\operatorname{dim}: R O(\mathbb{Z} / 2) \rightarrow \mathbb{Z}$, and the identification

$$
\mathcal{A}_{*}\left[\sigma^{ \pm 1}\right] /\left(\sigma^{-1}-1\right) \cong \mathcal{A}_{*} .
$$

Proof. By definition, the map $\rho$ is induced by $p_{+} \wedge i d_{H \mathbb{F}_{2} \wedge H \mathbb{F}_{2}}: \mathbb{Z} / 2_{+} \wedge H \underline{\mathbb{F}_{2}} \wedge H \underline{\mathbb{F}_{2}} \rightarrow$ $H \mathbb{F}_{2} \wedge H \mathbb{F}_{2}$, where $p: \mathbb{Z} / 2 \rightarrow *$ is the unique $\mathbb{Z} / 2$-set map. Now, $\rho$ induces a Hopf algebroid map because the restriction is induced by a ring $\mathbb{Z} / 2$-spectrum map $p_{+} \wedge$ $i d_{H \underline{\mathbb{F}_{2}} \wedge H \underline{\mathbb{F}_{2}}}$.

Remark 4.5. We choose to quotient out the ideal $\left(\sigma^{-1}-1\right)$. Another possibility would have been to choose $\left(\sigma^{-1}\right)$. The map would then have been the analogue for the dual Steenrod algebras of the map studied by Caruso $[\mathbf{6}]$.

\section{Quotients of the dual Steenrod algebra}

\subsection{Quotient Hopf algebroids}

Definition 5.1 (see A.1 $[\mathbf{1 5}]$ ). Let $(H, A)$ be an $R O(\mathbb{Z} / 2)$-graded Hopf algebroid. An ideal $I$ of $A$ is an $R O(\mathbb{Z} / 2)$-graded Hopf algebroid ideal if

$$
\begin{aligned}
\epsilon(I) & =0 \\
\Delta(I) & \subset I \otimes_{H} A \oplus A \otimes_{H} I \\
c(I) & \subset I .
\end{aligned}
$$

Proposition 5.2. Let $(H, A)$ be an $R O(\mathbb{Z} / 2)$-graded Hopf algebroid, and let $I$ be an $R O(\mathbb{Z} / 2)$-graded Hopf algebroid ideal of $(H, A)$. Then there is a natural $R O(\mathbb{Z} / 2)$ graded Hopf algebroid structure on $(H, A / I)$ such that the projection $A \rightarrow A / I$ induces an $R O(\mathbb{Z} / 2)$-graded Hopf algebroid morphism

$$
(H, A) \rightarrow(H, A / I) .
$$

Proof. The proof is an $R O(\mathbb{Z} / 2)$-graded version of the classical one.

Remark 5.3. Even when $A$ is $H$-free, since the ring $H$ is not a field, $A / I$ is not necessarily a free $H$-module.

Definition 5.4. An $R O(\mathbb{Z} / 2)$-graded Hopf algebroid $(H, A)$ is connected if $A$ is generated as an $H$-module in degrees $V \in R O(\mathbb{Z} / 2)$ such that $\operatorname{dim}(V) \geqslant 0$ with one generator of degree zero, which is a copy of $H$ generated by $\eta_{R}$ and $\eta_{L}$. 
Example 5.5. The $R O(\mathbb{Z} / 2)$-graded Hopf algebroid $\left(H \underline{\mathbb{F}}_{\star}{ }_{\star}^{\mathbb{Z} / 2}, \mathcal{A}_{\star}\right)$ is connected.

Proposition 5.6. If $(H, A)$ is connected and $I$ is an ideal of $A$ as an algebra satisfying

$$
\epsilon(I)=0
$$

and

$$
\Delta(I) \subset I \otimes_{H} A \oplus A \otimes_{H} I,
$$

then, $I$ is an $R O(\mathbb{Z} / 2)$-graded Hopf algebroid ideal.

Proof. The proof is analogous to the one in the classical $\mathbb{Z}$-graded case, reasoning with $\operatorname{dim}(|x|) \in \mathbb{Z}$ for the induction hypothesis.

\subsection{A family of quotient algebras of $\mathcal{A}_{\star}$}

In this subsection we define the particular quotient algebras of the equivariant Steenrod algebra we are interested in. The aim of the rest of this section is to develop a theory of profile functions for quotient Hopf algebroids of the $\mathbb{Z} / 2$-equivariant dual Steenrod algebra. The results we prove here for the $\mathbb{Z} / 2$-equivariant Steenrod algebra are analogous to those of Adams and Margolis [2]. The notation used here is very similar to that of Adams and Margolis for the non-equivariant odd primary case.

Definition 5.7. We call a profile function a pair of maps $(h, k)$,

$$
\begin{gathered}
h: \mathbb{N} \rightarrow \mathbb{N} \cup\{\infty\} \\
k: \mathbb{N} \backslash\{0\} \rightarrow \mathbb{N} \cup\{\infty\} .
\end{gathered}
$$

For profile functions $(h, k)$, denote by $I(h, k)$ the two-sided ideal of $\mathcal{A}_{\star}$ generated by $\xi_{i}^{2^{h(i)}}$ and $\tau_{i}^{k^{k(i)}}$ (with the convention $x^{\infty}=0$ ).

We think of profile functions $(h, k)$ as a way to encode the quotient algebra $\mathcal{A}_{\star} / I(h, k)$ of the $\mathbb{Z} / 2$-equivariant Steenrod algebra.

Example 5.8. Denote $h=(0, \infty, \infty, \ldots)$. We define two profile function which will serve as examples through the rest of this section:

- Let $I_{1}=I\left(h, k_{1}\right)$ for $k_{1}=(1,0, \infty, \infty, \ldots)$.

- Let $I_{2}=I\left(h, k_{2}\right)$ for $k_{2}=(\infty, 0, \infty, \infty, \ldots)$.

Because of the relation $\tau_{0}^{2}=a \tau_{1}+\eta_{R}\left(\sigma^{-1}\right) \xi_{1}$ in the $\mathbb{Z} / 2$-equivariant dual Steenrod algebra, the two ideals $I_{1}$ and $I_{2}$ coincide. In the next subsection, for a given ideal $I$ of $\mathcal{A}_{\star}$, we define a preferred choice of profile function defining $I$ called the minimal profile function.

\subsection{Minimality of profile functions}

Definition 5.9. - Define a partial order on profile functions $(h, k)$ by $(h, k) \leqslant$ $\left(h^{\prime}, k^{\prime}\right)$ if $\forall n \geqslant 0, h(n+1) \leqslant h^{\prime}(n+1)$ and $k(n) \leqslant k^{\prime}(n)$.

- We say that a profile function $(h, k)$ is minimal if it is minimal among the profile functions $\left(h^{\prime}, k^{\prime}\right)$ such that $I\left(h^{\prime}, k^{\prime}\right)=I(h, k)$. 
Lemma 5.1. 1. A profile function $(h, k)$ is minimal if and only if $\forall i, n \geqslant 0, \tau_{i}^{2^{n}} \in$ $I(h, k)$ is equivalent to $n \geqslant k(i)$.

2. Let $(h, k)$ be a profile function. Then the profile function $(h, \tilde{k})$ defined by

$$
\begin{aligned}
\tilde{k}: \mathbb{N} \backslash\{0\} & \rightarrow \mathbb{N} \cup\{\infty\} \\
n & \mapsto M i n\left\{l \in \mathbb{N} \mid \tau_{n}^{2^{l}} \in I(h, k)\right\} .
\end{aligned}
$$

is the unique minimal profile function such that $I(h, k)=I(h, \tilde{k})$.

Proof. 1. Let $(h, k)$ be a profile function. If $\tau_{i}^{2^{n}} \notin I(h, k)$, then $n \leqslant k(i)$; this proves $\Leftarrow$.

For $\Rightarrow$, let $(h, k)$ be a profile function. If $(h, k)$ do not satisfy the asserted hypothesis, there is an integer $i$ such that $\tau_{i}^{2^{k(i)-1}} \in I(h, k)$. Let $\tilde{k}(j)=\left\{\begin{array}{c}k(j) \text { if } j \neq i \\ k(i)-1 \text { if } i=j \text {. }\end{array}\right.$ Then we have $I(h, k)=I(h, \tilde{k})$ and $(h, \tilde{k})<(h, k)$. Thus, $(h, k)$ is not minimal.

2. First, observe that the two profile functions $(h, k)$ and $(h, \tilde{k})$ generates the same ideal. Moreover, by the first point, the profile function $(h, \tilde{k})$ is minimal. Thus, it remains to check that such a minimal couple is unique. It is a consequence of the first point: if $I(h, k)=I\left(h^{\prime}, k^{\prime}\right)$, then $h=h^{\prime}$, and the first point implies that for any profile function $\left(h^{\prime}, k^{\prime}\right)$ generating the ideal $I(h, k)$, we have $(h, \tilde{k}) \leqslant\left(h^{\prime}, k^{\prime}\right)$.

Example 5.10. Consider the examples defined in Example 5.8; the minimal profile function associated to $\left(h, k_{2}\right)$ is $\left(h, k_{1}\right)$. Indeed:

- The profile function $\left(h, k_{2}\right)$ is not minimal: $\tau_{0}^{2}=a \tau_{1}+\eta_{R}\left(\sigma^{-1}\right) \xi_{1} \in I(h, k)$ but $k_{2}(0)=\infty$, so the first point of Lemma 5.1 cannot be satisfied.

- The profile function $\left(h, k_{1}\right)$ is minimal.

\subsection{Conditions on the generating relations to provide a quotient Hopf algebroid}

We now focus on necessary and sufficient conditions on a profile function $(h, k)$ so that $\mathcal{A}_{\star} / I(h, k)$ has a Hopf algebroid structure induced by the quotient.

Remark 5.11. The following should be compared to the odd primary case of [2]. The similarity between the two cases comes from the fact that the formulas for the coproduct in $\mathcal{A}_{*}^{p}$ for odd $p$ (with the notations of [2]) and $\mathcal{A}_{\star}$ (with our notations) are the same.

Proposition 5.12. Let $(h, k)$ be a minimal pair of profile functions. The ideal $I(h, k)$ is an $R O(\mathbb{Z} / 2)$-graded Hopf algebroid ideal if and only if the profile function satisfy

$$
\begin{array}{r}
\forall i, j \geqslant 1, h(i) \leqslant j+h(i+j) \text { or } h(j) \leqslant h(i+j) \\
\forall i \geqslant 1, j \geqslant 0, h(i) \leqslant j+k(i+j) \text { or } k(j) \leqslant k(i+j) .
\end{array}
$$

Proof. We check the equivalence between the three conditions of Definition 5.1 and Equations (2) and (3).

By definition of $I(h, k), \epsilon(I(h, k))=0$. Moreover, Proposition 5.6 applies to the pair $\left(H{\underline{F_{2}}}_{\star}^{\mathbb{Z} / 2}, \mathcal{A}_{\star} / I(h, k)\right)$, giving that $c(I(h, k)) \subset I(h, k)$ is automatically satisfied if $\Delta(I(h, k)) \subset I(h, k) \otimes \mathcal{A}_{\star} \oplus \mathcal{A}_{\star} \otimes I(h, k)$. 
Thus, it suffices to show that $\Delta(I(h, k)) \subset I(h, k) \otimes \mathcal{A}_{\star} \oplus \mathcal{A}_{\star} \otimes I(h, k)$ is equivalent to

$$
\begin{array}{r}
\forall i, j \geqslant 1, h(i) \leqslant j+h(i+j) \text { or } h(j) \leqslant h(i+j) \\
\forall i \geqslant 1, j \geqslant 0, h(i) \leqslant j+k(i+j) \text { or } k(j) \leqslant k(i+j) .
\end{array}
$$

We check that the conditions are equivalent, using the known structure of the $\mathbb{Z} / 2$-equivariant dual Steenrod algebra. The coproduct $\Delta$ is an algebra morphism, so $I(h, k)$ satisfies $\Delta(I(h, k)) \subset I(h, k) \otimes \mathcal{A}_{\star} \oplus \mathcal{A}_{\star} \otimes I(h, k)$ if and only if, for all $i \geqslant 1$, $\Delta\left(\xi_{i}^{2^{h(i)}}\right) \in I(h, k) \otimes \mathcal{A}_{\star} \oplus \mathcal{A}_{\star} \otimes I(h, k)$ and, for all $i \geqslant 0, \Delta\left(\tau_{i}^{2^{k(i)}}\right) \in I(h, k) \otimes \mathcal{A}_{\star} \oplus$ $\mathcal{A}_{\star} \otimes I(h, k)$. But

$$
\Delta\left(\xi_{n}^{2^{h(n)}}\right)=\sum_{i+j=n} \xi_{i}^{2^{j+h(n)}} \otimes \xi_{j}^{2^{h(n)}}
$$

and

$$
\Delta\left(\tau_{n}^{2^{k(n)}}\right)=\sum_{i+j=n} \xi_{i}^{2^{j+k(n)}} \otimes \tau_{j}^{2^{k(n)}} .
$$

By the first equation, $\Delta\left(\xi_{i}^{2^{h(i)}}\right) \in I(h, k) \otimes \mathcal{A}_{\star} \oplus \mathcal{A}_{\star} \otimes I(h, k)$ is equivalent to 4 . For the second one, $\tau_{n}^{2^{k(n)}} \in I(h, k)$ by definition of $I(h, k)$. Now, for all $i, j$ such that $i+$ $j=n$, either $\xi_{i}^{2^{j+k(n)}} \in I(h, k)$ or $\tau_{j}^{2^{k(n)}} \in I(h, k)$. By minimality, the second condition is equivalent to $k(j) \leqslant k(n)$.

\subsection{Freeness of quotient Hopf algebroids}

We now address the problem observed in Remark 5.3. We will give a numerical condition on a pair of profile functions $(h, k)$ to ensure that the associated quotient algebra $\mathcal{A}_{\star} / I(h, k)$ is free over $H \underline{\mathbb{F}}_{\star} \underset{ }{\mathbb{Z} / 2}$.

Definition 5.13. We say that a pair of profile functions $(h, k)$ is free if it satisfies the following property: $\forall i \geqslant 0, m \geqslant k(i), j \leqslant m$,

$$
k(i+m)=0,
$$

and

$$
h(i+j) \leqslant m-j .
$$

Lemma 5.2. Let $(h, k)$ be a free pair of profile functions. Then, for all $i \geqslant 0$ and $m \geqslant 0$, the following conditions are equivalent:

1. $\tau_{i}^{2^{m}} \in I(h, k)$.

2. In the decomposition of $\tau_{i}^{2^{m}}$ in the monomial basis-i.e., $\tau_{i}^{2^{m}}=\sum_{j} h_{j} x_{j}$-for some $h_{j} \in H \underline{\mathbb{F}}_{\star}{ }_{\star}^{\mathbb{Z} / 2}$ and $x_{j} \in \mathcal{B}_{m}$, we have that if $j \geqslant 0$, then $x_{j} \in I(h, k)$.

3. $\tau_{i+m} \in I(h, k)$ and $\forall 0 \leqslant j \leqslant m-1, \xi_{i+m-j}^{2^{j}} \in I(h, k)$.

Proof. $2 \Leftrightarrow 3$ : Write $\tau_{i}^{2^{m}}$ in the monomial basis. By the relation $\tau_{i}^{2}=a \tau_{i+1}+\eta_{R}\left(\sigma^{-1}\right)$ 
$\xi_{i+1}$ in the $\mathbb{Z} / 2$-equivariant dual Steenrod algebra, thus

$$
\tau_{i}^{2^{m}}=a^{2^{m-1}} \tau_{i+1}^{2^{m-1}}+\eta_{R}\left(\sigma^{-1}\right)^{2^{m-1}} \xi_{i+1}^{2^{m-1}} .
$$

By induction on $m$, we find

$$
\tau_{i}^{2^{m}}=a^{2^{m}-1} \tau_{i+m}+\sum_{j=1}^{m} a^{2^{m}-2^{j}} \eta_{R}\left(\sigma^{-1}\right)^{2^{j-1}} \xi_{i+m-j+1}^{2^{j}},
$$

and the result follows.

$2 \Rightarrow 1$ : Suppose 2 . Then equation (6) implies that $\tau_{i}^{2^{m}} \in I(h, k)$.

$1 \Rightarrow 2$ : If $\tau_{i}^{2^{m}} \in I(h, k)$, suppose that the implication is true for all $m^{\prime}<m$ and $i^{\prime}<i$. Then one of the following assertions is satisfied:

- $m \geqslant k(i)$, and thus freeness of $(h, k)$ implies that every element of the monomial basis appearing in Equation (6) are already in $I(h, k)$.

- $m<k(i)$. In that case, $a^{2^{m-1}} \tau_{i+1}^{2^{m-1}}+\eta_{R}\left(\sigma^{-1}\right)^{2^{m-1}} \xi_{i+1}^{2^{m-1}} \in I(h, k)$. The ideal $I(h, k)$ is generated by monomials, so $\tau_{i+1}^{2^{m-1}} \in I(h, k)$ and $\eta_{R}\left(\sigma^{-1}\right)^{2^{m-1}} \xi_{i+1}^{2^{m-1}} \in$ $I(h, k)$. The powers of the elements $\tau_{i^{\prime}}^{m^{\prime}}$ appearing in this expression satisfies $m^{\prime}<m$ and $i^{\prime}<i$, so their decomposition in the monomial basis consists only in terms of $I(h, k)$ by the induction hypothesis.

In each case, by the induction step holds, concluding the proof.

Proposition 5.14. If $(h, k)$ is a free pair of profile functions, then the $H{\underline{\mathbb{F}_{2}}}_{\star}^{\mathbb{Z} / 2}$ module $\mathcal{A}_{\star} / I(h, k)$ is free. Moreover, a basis for this $H_{\mathbb{F}_{2}}^{\mathbb{Z} / 2}$-module is

$$
\mathcal{B}_{(h, k)}=\left\{\left[b_{i}\right] \mid b_{i} \in \mathcal{B}_{m}, b_{i} \notin I(h, k)\right\},
$$

or, equivalently, consists in elements of the monomial basis that are of the form

$$
\Pi_{i \geqslant 0} \xi_{i+1}^{h_{i}} \tau_{i}^{\epsilon_{i}}
$$

where $\forall i \geqslant 0, \epsilon_{i} \leqslant 2^{k(i)}$ and $h_{i+1} \leqslant 2^{h(i+1)}, \epsilon_{i}=0$ or 1 , and the product is finite.

Proof. Consider the canonical $\mathrm{HF}_{\mathbb{F}_{\star}}{ }_{\star}^{\mathbb{Z} / 2}$-module morphism

$$
\phi: H{\underline{\mathbb{F}_{2}}}_{\star}^{\mathbb{Z} / 2}\left\{\mathcal{B}_{(h, k)}\right\} \rightarrow \mathcal{A}_{\star} / I(h, k) .
$$

Suppose that $(h, k)$ is free; we will show that $\phi$ is a $H \underline{\mathbb{F}}_{2} \mathbb{Z} / 2$-module isomorphism.

Let $x \in \operatorname{Ker}(\phi)$, i.e., $x=\sum h_{i} b_{i}$ for $h_{i} \in H{\underline{\mathbb{F}_{2}}}_{\star}{ }_{\star} / 2$ and $b_{i} \in \mathcal{B}_{(h, k)}$ such that $x \in$ $I(h, k)$. Consequently, there exists $x_{i}, y_{i} \in \mathcal{A}_{\star}$ such $^{\star}$ that

$$
x=\sum_{i \geqslant 0} x_{i} \xi_{i}^{2^{h(i)}}+y_{i} \tau_{i}^{2^{k(i)}} .
$$

Thus for all $i \geqslant 0$,

- each element of the monomial basis appearing in the decomposition of $x_{i} \xi_{i}^{2^{h(i)}}$ is an element of $I(h, k)$ because $\xi_{i}^{2^{h(i)}} \in I(h, k)$, and

- by Lemma 5.2, each element of the monomial basis appearing in the decomposition of $\tau_{i}^{2^{k(i)}}$ is in $I(h, k)$, so the same is true for each element of the monomial basis appearing in the decomposition of $y_{i} \tau_{i}^{2^{k(i)}}$. 
Finally we write $x \in H \underline{\mathbb{F}}_{\star} \underset{ }{\mathbb{Z} / 2}\left\{\mathcal{B}_{(h, k)}\right\} \cap H \underline{\mathbb{F}}_{\star}{ }_{\star}^{\mathbb{Z} / 2}\left\{\mathcal{B}_{m} \backslash \mathcal{B}_{(h, k)}\right\}$; thus $x=0$.

\section{Notable examples and properties}

\subsection{Some examples of quotient Hopf algebroids of $\mathcal{A}_{\star}$}

Definition 6.1. For a map $h: \mathbb{N}-\{0\} \rightarrow \mathbb{N} \cup\{\infty\}$, we denote by $J(h)$ the ideal of the modulo 2 non-equivariant Steenrod algebra generated by the elements $\xi_{i}^{2^{h(i)}}$.

Remark 6.2. The result [2, Theorem 3.3] gives a necessary and sufficient condition on $h$ such that $J(h)$ is a Hopf algebra ideal.

Proposition 6.3. There is an injection of partially ordered sets

$\left\{\right.$ quotient Hopf algebras of $\left.\mathcal{A}_{*}^{2}\right\} \rightarrow\left\{\right.$ quotient Hopf algebroids of $\left.\mathcal{A}_{\star}\right\}$

$$
\mathcal{A}_{*}^{2} / J(h) \mapsto \mathcal{A}_{\star} / I(h, 0)
$$

where the partial order is induced by inclusion of ideals.

Proof. Let $\mathcal{B}_{*}$ be a Hopf algebra quotient of $\mathcal{A}_{*}^{2}$. By the main result of [2], we know that there exists a unique $h: \mathbb{N} \backslash\{0\} \rightarrow \mathbb{N} \cup\{\infty\}$ satisfying the conditions of Proposition 5.12 for $k=0$ (the second one becomes trivial for $k=0$, and the first one becomes precisely the one asserted by $[\mathbf{2}])$ such that $\mathcal{B}_{*}$ is isomorphic to $\mathcal{A}_{*}^{2} / J(h)$.

Thus, Proposition 5.12 implies that the application is well defined. The fact that it preserves the partial order is true by construction.

The previous proposition allows us to define some examples of quotient Hopf algebroids, and, by duality (Proposition 3.12 under the Hypothesis 3.10 that is satisfied by Proposition 4.3); this provides some particular subalgebras of the $\mathbb{Z} / 2$-equivariant Steenrod algebra.

Definition 6.4. Define $\widetilde{\mathcal{A}(n)_{\star}}=\mathcal{A}_{\star} / I\left(h_{n}, 0\right)$ for $h_{n}=(n-1, n-2, n-3, \ldots, 0,0 \ldots)$. And denote $\widehat{\mathcal{A}(n)^{\star}}=\operatorname{Hom}_{H \underline{\mathbb{F}}_{\star}}^{\mathbb{Z} / 2}\left(\widehat{\left(\mathcal{A}(n)_{\star}\right.}, H \underline{\mathbb{F} 2}_{\star}^{\mathbb{Z} / 2}\right)$ their dual algebras.

These algebras are analogous to the squares of the classical $\mathcal{A}(n)^{*} \subset \mathcal{A}_{2}^{*}$. We now define genuine analogues to the classical sub-Hopf algebras of $\mathcal{A}_{2}^{*} \mathcal{A}^{\star}(n)$ and $\mathcal{E}^{\star}(n)$.

\section{Definition 6.5.}

- Let $\mathcal{E}(n)_{\star}=\mathcal{A}_{\star} / I\left(0, k_{n}\right)$ for $k_{n}=(n, n-1, n-2, \ldots, 0,0, \ldots)$. Denote by $\mathcal{E}(\infty)_{\star}$ the quotient Hopf algebroid $\mathcal{A}_{\star} / I(0, k)$ for $k=(\infty, \infty, \infty, \ldots)$, and by $\mathcal{E}^{\star}(n)$ and $\mathcal{E}^{\star}(\infty)$ their dual algebras.

- Let $\mathcal{A}(n)_{\star}=\mathcal{A}_{\star} / I\left(h_{n}, k_{n}\right)$ for $h_{n}=(n-1, n-2, n-3, \ldots, 0,0 \ldots)$ and $k_{n}=$ $(n, n-1, n-2, \ldots, 0,0, \ldots)$, and denote by $\mathcal{A}(n)^{\star}$ its dual algebra.

Remark 6.6. Proposition 5.14 and the fact that the profile functions defining the $\mathcal{E}(\infty)_{\star}, \mathcal{E}(n)_{\star}$, and $\mathcal{A}(n)_{\star}$ are free implies that these are free $H \underline{F}_{2}{ }_{\star} \mathbb{Z} / 2$-modules, with basis $\tau_{0}^{\epsilon_{0}}, \ldots \tau_{n}^{\epsilon_{n}}$, for some $\epsilon_{i} \in\{0,1\}\left(n=\infty\right.$ for $\left.\mathcal{E}(\infty)_{\star}\right)$. 


\subsection{Cofreeness $\left(H \underline{\mathbb{F}}_{2_{\star}}^{\mathbb{Z} / 2}, \mathcal{A}_{\star}\right)$ over its quotients}

We now answer the question of cofreeness of the various quotient Hopf algebroids of $\left(H \mathbb{F}_{2}{ }_{\star}^{\mathbb{Z} / 2}, \mathcal{A}_{\star}\right)$ over each other. It turns out to have a very general answer when modules are concentrated in positive twists. We first define a version of the $\mathbb{Z} / 2$ equivariant dual Steenrod algebra concentrated in positive twists.

Definition 6.7. Let $(H \geqslant, \mathcal{A} \geqslant)$ be the Hopf algebroid

$$
\left(\mathbb{F}_{2}\left[a, \sigma^{-1}\right], \mathbb{F}_{2}\left[a, \sigma^{-1}\right]\left[\tau_{i}, \xi_{i}+1, i \geqslant 0\right] / \tau_{i}^{2}=a \tau_{i+1}+\eta_{R}\left(\sigma^{-1}\right) \xi_{i+1}\right)
$$

with the same formulae as for the $\mathbb{Z} / 2$-equivariant dual Steenrod algebra for the structure maps, that is,

$$
\begin{aligned}
& \Delta\left(\xi_{n}\right)=\sum_{i=0}^{n} \xi_{n-i}^{p^{i}} \otimes \xi_{i} \\
& \Delta\left(\tau_{n}\right)=\sum_{i=0}^{n} \xi_{n-i}^{p^{i}} \otimes \tau_{i}+\tau_{n} \otimes 1 .
\end{aligned}
$$

We now recall the construction of the extension of objects for a small groupoid.

Definition 6.8. Let $\mathcal{O}$ be the set of objects, and let $\mathcal{M}$ be the set of morphisms of a small groupoid. A a left $(\mathcal{O}, \mathcal{M})$-module structure on a set $\mathcal{Y}$ over $\mathcal{O}$ is a map

$$
\psi: \mathcal{M} \times_{\mathcal{O}} \mathcal{Y} \rightarrow \mathcal{Y}
$$

where $\mathcal{M}$ is seen as a set over $\mathcal{O}$ via the source morphism which is compatible with projection, associative and unital.

Proposition 6.9. Let $\mathcal{Y}$ be a left $(\mathcal{O}, \mathcal{M})$-module. Then there is a natural groupoid structure on $\left(\mathcal{Y}, \mathcal{M} \times_{\mathcal{O}} \mathcal{Y}\right)$ defined by:

1. identity: $\mathcal{Y}=\mathcal{O} \times_{\mathcal{O}} \mathcal{Y} \stackrel{e \times \mathcal{Y}}{\longrightarrow} \mathcal{M} \times_{\mathcal{O}} \mathcal{Y}$ where $e$ is the identity,

2. source: $\operatorname{pr}_{\mathcal{Y}}$,

3. target: $\psi$,

4. inverse: $\operatorname{pr}_{\mathcal{M}} \times_{\mathcal{O}} \psi: \mathcal{M} \times_{\mathcal{O}} \mathcal{Y} \rightarrow \mathcal{M} \times_{\mathcal{O}} \mathcal{Y}$ where $c$ is the inverse in the groupoid, and

5. composition: $\circ \times \mathcal{Y}: \mathcal{M} \times_{\mathcal{O}} \mathcal{Y} \times \mathcal{Y} \mathcal{M} \times_{\mathcal{O}} \mathcal{Y} \rightarrow \mathcal{M} \times_{\mathcal{O}} \mathcal{Y}$

Proof. It suffices to check the axioms of a groupoid one by one.

Lemma 6.1. The algebra $H \underline{\mathbb{F}}_{\star}^{\mathbb{Z} / 2}$ is an $\left(H_{\geqslant}, \mathcal{A}_{\geqslant}\right)$-comodule algebra, via the left unit; i.e., $\psi(a)=a \otimes 1, \psi\left(\sigma^{-1}\right)=\sigma^{-1} \otimes 1+a \otimes \tau_{0}$, and $\psi\left(\sigma^{2}\right)=\sigma^{2} \otimes 1$.

Proof. This is an immediate consequence of the formulae defining the product and coproduct in $\left(H_{\geqslant}, \mathcal{A}_{\geqslant}\right)$.

Proposition 6.10. The Hopf algebroid $\left(H_{\geqslant}, \mathcal{A} \geqslant\right)$ satisfies the following properties.

1. It is a deformation of the non-equivariant dual modulo 2 Steenrod algebra, with $\mathfrak{m}=\left(a, 1-\sigma^{-1}\right)$.

2. The Hopf algebroid $\left(H \underline{\mathbb{F}}_{\star} \underset{\star}{\mathbb{Z} / 2}, \mathcal{A}_{\star}\right)$ is isomorphic to $H \underline{\mathbb{F}}_{\star}{ }_{\star}^{\mathbb{Z} / 2} \otimes_{H \geqslant}\left(H \geqslant, \mathcal{A}_{\geqslant}\right)$. 
Proof. For the first point, observe that by definition of the structure morphisms:

- on objects,

$$
H_{\geqslant} / \mathfrak{m}=\mathbb{F}_{2}
$$

- on morphisms, for the generators as $H_{\geqslant}$-algebra,

$$
\begin{aligned}
\mathcal{A}_{\geqslant} / \mathfrak{m} & \rightarrow \mathcal{A}_{*} \\
\xi_{i} & \mapsto \xi_{i}^{2} \\
\tau_{i} & \mapsto \xi_{i} .
\end{aligned}
$$

For the second point, since a Hopf algebroid is a cogroupoid object in the category of algebras, by naturality of the construction of Proposition 6.9 and because a comodule algebra corepresents a functor whose values are left modules over the groupoid corresponding to the Hopf algebroid, Proposition 6.9 provides a Hopf alge-

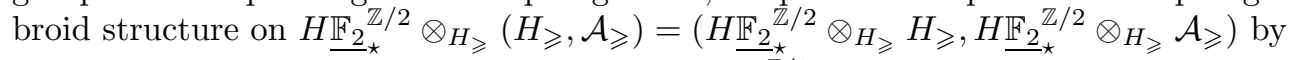
the $(H \geqslant, \mathcal{A} \geqslant)$-comodule algebra structure on $\underline{H \underline{F}}_{\underline{F}_{\star}}^{\mathbb{Z}} / 2$ of Lemma 6.1 . The assertion is then a consequence of the definitions.

\subsection{Cofreeness}

In this subsection, all algebras and Hopf algebroids are implicitly finite-dimensional $\mathbb{F}_{2}$-vector spaces.

The goal of this subsection is to understand when an $R O(\mathbb{Z} / 2)$-graded quotient Hopf algebroid of $(H, A)$ satisfies that $(H, A)$ is free as a comodule over it.

Remark 6.11. In the non-equivariant case, $\mathcal{A}_{*}$ is cofree as a comodule over all its quotient Hopf algebras (see [14, Proposition 4.4]). For Hopf algebroids, the situation is more tricky. We will use a modified version of [15, Theorem A.1.1.17] (Comodule Algebra Structure Theorem) to understand the cofreeness of an $R O(\mathbb{Z} / 2)$-graded quotient Hopf algebroid over its quotients.

We now study aspects of deformation theory for Hopf algebroids (we make a slight abuse of terminology by talking about deformation in the context of augmented graded rings, and not of complete local rings). The motivation comes from Proposition 4.4, and the previous remark.

Definition 6.12. A deformation of an $R O(\mathbb{Z} / 2)$-graded connected Hopf algebroid $(H, A)$ is an $R O(\mathbb{Z} / 2)$-graded connected Hopf algebroid $(\tilde{H}, \tilde{A})$ together with an ideal $\mathfrak{m} \subset H$ concentrated in degrees of the form $*+n \alpha$ for $n$ non-negative, and an identification $\left(\tilde{H} / \mathfrak{m}, \tilde{A} / \tilde{A} \eta_{L}(\mathfrak{m})\right) \cong(H, A)$. More precisely:

1. $\tilde{H} / \mathfrak{m} \cong H$.

2. Via the unit $\eta_{L}$, we can consider the left ideal generated by $\mathfrak{m}$ in $\tilde{A}$. Suppose that $\tilde{A} / \tilde{A} \eta_{L}(\mathfrak{m}) \cong A$.

3. These two isomorphisms induce an isomorphism of Hopf algebroid.

Theorem 6.13. Let $(\tilde{H}, \tilde{A})$ be an $R O(\mathbb{Z} / 2)$-graded connected Hopf algebroid, and let $\tilde{\mathcal{I}_{B}} \subset \tilde{\mathcal{I}_{C}}$ be two $R O(\mathbb{Z} / 2)$-graded Hopf algebroid ideals of $(\tilde{H}, \tilde{A})$. We use the following 
notation:

$$
\begin{aligned}
\tilde{B} & =\tilde{A} / \tilde{\mathcal{I}_{B}} \\
\tilde{C} & =\tilde{A} / \tilde{\mathcal{I}_{C}} \\
\mathcal{I}_{B} & =\tilde{\mathcal{I}_{B}} / \tilde{\mathcal{I}_{B}} \cap \tilde{A} \eta_{L}(\mathfrak{m}) \\
\mathcal{I}_{C} & =\tilde{\mathcal{I}_{C}} / \tilde{\mathcal{I}_{C}} \cap \tilde{A} \eta_{L}(\mathfrak{m}) \\
B & =A / \mathcal{I}_{B} \\
C & =A / \mathcal{I}_{C} .
\end{aligned}
$$

Suppose, moreover, that:

1. The ideals $\mathcal{I}_{B}$ and $\mathcal{I}_{C}$ are Hopf algebroid ideals of $A$. Thus, $(H, B)$ and $(H, C)$ are Hopf algebroids, and there are Hopf algebroid morphisms $A \rightarrow B$ and $A \rightarrow$ C.

2. The map $B=A / \mathcal{I}_{B} \rightarrow A / \mathcal{I}_{C}=C$ makes $B$ a cofree $C$-comodule.

3. $\tilde{B}$ and $\tilde{C}$ are free $H$-modules.

Then $\tilde{B}$ is a cofree $\tilde{C}$-comodule.

Proof. This proof is an appropriately modified version of [15, Theorem A1.1.17]. The $C$-comodule $B$ is cofree, so let $\left\{1, b_{i}\right\}$ be a basis of $B$ as a counital $C$-comodule, and denote $\psi: B \rightarrow C \otimes_{H} H\left\{1, b_{i}\right\}$ the $C$-comodule isomorphism such that $B \rightarrow C$ is the $C$-comodule map such that $b_{i} \mapsto 0$.

By hypothesis, $\tilde{C}$ is a free $H$-module. Choose a basis $\left\{1, y_{i}\right\}$ of $\tilde{C}$ as an $\tilde{H}$-module, where 1 comes from $\eta_{L}$. The set $\left\{x_{i}\right\}=\left\{1, y_{j}\right\} \times\left\{1, b_{k}\right\}$ is then a basis of $\tilde{B}$ as an $\tilde{H}$-module.

We also have the following identification

$$
\begin{aligned}
B & \cong \tilde{B} / \tilde{B}(\mathfrak{m}) \\
& \cong \tilde{H}\left\{x_{i}\right\} /\left((\mathfrak{m}) \otimes_{\mathbb{F}_{2}} \mathbb{F}_{2}\left\{x_{i}\right\}\right) \\
& \cong \tilde{H} /((\mathfrak{m})) \otimes_{\mathbb{F}_{2}} \mathbb{F}_{2}\left\{x_{i}\right\} \\
& \cong H\left\{x_{i}\right\} .
\end{aligned}
$$

thus, $\left\{x_{i}\right\}$ is also an $H$-module basis for $B$. Finally, the $\tilde{H}$-module morphism

$$
\tilde{\psi}: \tilde{B} \rightarrow \tilde{H}\left\{1, b_{i}\right\}
$$

induced by $y_{i} \mapsto 0$ extends $(\epsilon \otimes 1) \circ \psi: B \rightarrow H\left\{1, b_{i}\right\}$.

Let $\tilde{\phi}$ be the graded $\tilde{H}$-module morphism defined as the composite

$$
\tilde{B} \stackrel{\Delta}{\rightarrow} \tilde{B} \otimes_{\tilde{H}} \tilde{B} \rightarrow \tilde{C} \otimes_{\tilde{H}} \tilde{B} \stackrel{i d \otimes \tilde{\psi}}{\longrightarrow} \tilde{C} \otimes_{\tilde{H}} \tilde{H}\left\{1, b_{i}\right\} .
$$

And define $\phi$ as the $C$-comodule morphism

$$
\phi: B \stackrel{\Delta}{\rightarrow} B \otimes_{H} B \rightarrow C \otimes_{H} B \stackrel{i d \otimes((\epsilon \otimes i d) \circ \psi)}{\longrightarrow} C \otimes_{H} H\left\{b_{i}\right\} .
$$

The map $\phi$ is an isomorphism. Moreover, $\psi$ is a $C$-comodule isomorphism, and projection is compatible with the coproduct on

$$
B \rightarrow B \otimes_{H} B
$$

and

$$
C \rightarrow C \otimes_{H} C
$$


so $\phi$ is also a $C$-comodule isomorphism.

We now show that $\tilde{\phi}$ is an isomorphism. The strategy goes as follows: We define two decreasing filtrations on $\tilde{B}$ and $\tilde{C} \otimes_{\tilde{H}} \tilde{H}\left\{b_{i}\right\}$ which are compatible with the map $\tilde{\phi}$. We then show that $\tilde{\phi}$ induces an isomorphism on the graded object associated with this filtration.

The filtrations are defined on $\tilde{C} \otimes_{\tilde{H}} \tilde{H}\left\{b_{i}\right\}$ and $\tilde{B}$ by

$$
\begin{aligned}
F^{d}\left(\tilde{C} \otimes_{\tilde{H}} \tilde{H}\left\{b_{i}\right\}\right) & =\left\langle c \otimes h b_{i} \mid \operatorname{deg}\left(h b_{i}\right) \geqslant d\right\rangle \\
F^{d}(\tilde{B}) & =\widetilde{\phi}^{-1}\left(F^{d}\left(\tilde{C} \otimes_{\tilde{H}} \tilde{H}\left\{b_{i}\right\}\right)\right),
\end{aligned}
$$

where $\langle S\rangle$, for a set $S$ stands for the cofree $\tilde{C}$-comodule on $S$. We check that $\tilde{\phi}$ is an isomorphism on the graded object associated with this filtration.

Write $\Delta(b)=\sum b^{\prime} \otimes b^{\prime \prime}$ for $b$ in $\tilde{B}$. By definition of $\tilde{\phi}$, we have

$$
\tilde{\phi}(b)=\sum\left[b^{\prime}\right] \otimes \tilde{\psi}\left(b^{\prime \prime}\right) .
$$

So, let $\tilde{b}$ be a homogeneous element of $\tilde{B}$, and write $\tilde{B}$ in the basis $\left\{x_{i}\right\}$

$$
\tilde{b}=\sum h_{i} x_{i} .
$$

Consider first an element of the form $h_{i} x_{i}$. By definition of Hopf algebroid deformation, $\mathfrak{m}$ is concentrated in degrees of non-negative twists. Thus, the equality $\Delta_{\tilde{B}}=$ $\Delta_{B}$ modulo $\mathfrak{m}$ gives

$$
\Delta_{\tilde{B}}\left(h_{i} b_{i}\right) \equiv h_{i} \Delta_{\tilde{B}}\left(b_{i}\right) \equiv h_{i} \Delta_{B}\left(b_{i}\right)(\bmod \text { terms of lower twist }) \text {; }
$$

thus, on the graded object associated to the filtration $F^{\bullet}$, the morphism $\tilde{\phi}$ coincides with the unique $\tilde{H}$-module morphism that extends $\phi$.

Now define a filtration $F^{\bullet}$ on $C \otimes_{H} H\left\{b_{i}\right\}$ by

$$
F^{d}\left(C \otimes_{H} H\left\{b_{i}\right\}\right)=<c \otimes h b_{i} \mid \operatorname{deg}\left(h b_{i}\right) \geqslant d>
$$

and one on $B$ by pulling back along $\phi$.

The map $\phi$ being an isomorphism, it induces an isomorphism on the associated graded $C \otimes_{H} H\left\{b_{i}\right\}$ by the filtration $F^{d}$. But $\tilde{\phi}$ coincides with $\phi$ on the graded object associated to $F^{\bullet}$. We conclude that $\tilde{\phi}$ induces an isomorphism on the graded object associated with the filtration $F^{\bullet}$. Finally, the filtration is exhaustive, and the finitedimensional hypothesis in each degree allows us to apply the Mittag-Leffler criterion to conclude that the filtration is also complete. Consequently, $\tilde{\phi}$ is a $\tilde{C}$-comodule isomorphism.

Theorem 6.14. Let $B$ and $C$ be two quotient $R O(\mathbb{Z} / 2)$-graded Hopf algebroids of $\left(H \underline{F}_{\star}{ }_{\star}^{\mathbb{Z} / 2}, \mathcal{A}_{\star}\right)$ defined by two profile functions $\left(h_{B}, k_{B}\right) \geqslant\left(h_{C}, k_{C}\right)$. Then there is an

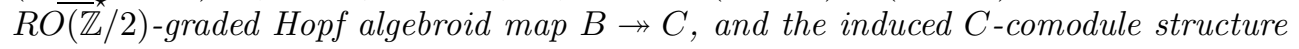
on $B$ is cofree.

Proof. We use that the non-equivariant modulo 2 dual Steenrod algebra is cofree over all its Hopf algebra quotients; thus the hypotheses of Proposition 6.13 are satisfied for the deformation given in Proposition 6.10. The result is then a consequence of Theorem 6.13. 
Corollary 6.15. For all $n \geqslant 0$, the Hopf algebroid $\left(\mathrm{HF}_{\underline{F}_{\star}}{ }_{\star}^{\mathbb{Z} / 2}, \mathcal{A}_{\star}\right)$ is cofree as a comodule over its quotients $\mathcal{E}(n)_{\star}$ and $\mathcal{A}(n)_{\star}$, and these quotient Hopf algebroids are cofree over each other.

Theorem 6.16. For all $n \geqslant 0$, the $\mathbb{Z} / 2$-equivariant Steenrod algebra $H \underline{\mathbb{F}}_{2}{ }_{\mathbb{Z} / 2} H \mathbb{F}_{2}$ is free as a module over $\mathcal{E}(n)^{\star}$ and $\mathcal{A}(n)^{\star}$, and these algebras are free over each other.

Proof. Because of Corollary 4.3, Proposition 3.12 is satisfied for $E=H \mathbb{F}_{2}$. Thus, Boardman's duality theorem (cf. Proposition 3.7) provides an equivalence between modules over the $\mathbb{Z} / 2$-equivariant Steenrod algebra and comodules over $\left(H \underline{\mathbb{F}}_{2} \underset{\mathbb{Z}}{ }{ }_{\star}, 2, \mathcal{A}_{\star}\right)$. This is now a consequence of Corollary 6.15.

As a consequence of this result, we have an easy computation in K-theory with reality. We first recall a possible construction of this $\mathbb{Z} / 2$-equivariant cohomology theory, following Atiyah [3]

Definition 6.17. Let $X$ be a $\mathbb{Z} / 2$-space. A Real vector bundle over $X$ is a complex vector bundle over $X$ such that the action of $\mathbb{Z} / 2$ is anti-linear on the fibers.

The following proposition is due to Atiyah $[\mathbf{3}]$.

Proposition 6.18. The functor $K \mathbb{R}_{0}$, defined on objects by $K \mathbb{R}^{0}(X)=G r(\operatorname{Vect} \mathbb{R}(X))$, extends to a cohomology theory. Denote by $K \mathbb{R}$ the corresponding $\mathbb{Z} / 2$-spectrum.

Corollary 6.19 (Corollary of Theorem 6.16). One has an isomorphism of $\mathbb{F}_{2}[a]$ modules

$$
H \underline{\mathbb{F}}_{2}^{\star} / 2 k \mathbb{R} \cong \mathcal{A}^{\star} / \mathcal{E}^{\star}(2) .
$$

Proof. The proof is similar to the computation of $H \mathbb{F}_{2}^{*}(k u)$, in two steps.

1. First compute $H \mathbb{F}_{2}^{*}(H \mathbb{Z})$ by the Bockstein spectral sequence associated to the exact couple

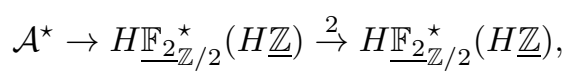

which collapses by freeness of $\mathcal{A}^{\star}$ over $\mathcal{E}(1)^{\star}$, giving $H \underline{\mathbb{F}_{2}}{ }_{\mathbb{Z} / 2}(H \underline{\mathbb{Z}}) \cong \mathcal{A}^{\star} / \mathcal{E}(1)^{\star}$.

2. Then the Bockstein spectral sequence associated to the exact couple

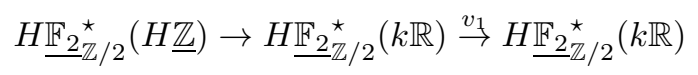

collapses by freeness of $\mathcal{A}^{\star}$ over $\mathcal{E}(2)^{\star}$. The result follows.

Remark 6.20. Another use of Theorem 6.14 is to provide change of rings isomorphisms for the $E^{2}$ page of the $\mathbb{Z} / 2$-equivariant Adams spectral sequence (cf. [10, Corollary 6.47]) that converges to cohomology with respect to spectra whose homology is of the form $\mathcal{A}_{\star} / / \mathcal{B}$, for $\mathcal{B}=\mathcal{A}(n)_{\star}$ or $\mathcal{E}(n)_{\star}$. 


\section{References}

[1] J.F. Adams, Stable homotopy and generalised homology, Chicago Lectures in Mathematics, University of Chicago Press, Chicago, IL, 1995, Reprint of the 1974 original.

[2] J.F. Adams and H.R. Margolis, Sub-Hopf-algebras of the Steenrod algebra, Proc. Cambridge Philos. Soc. 76 (1974), 45-52.

[3] M.F. Atiyah, K-theory and reality, Quart. J. Math. Oxford Ser. (2) 17 (1966), $367-386$.

[4] J.M. Boardman, Stable operations in generalized cohomology, In Handbook of algebraic topology. North-Holland, Amsterdam, 1995, pp. 585-686.

[5] R.R. Bruner and J.P.C. Greenlees, Connective real $K$-theory of finite groups, vol. 169 of Mathematical Surveys and Monographs, American Mathematical Society, Providence, RI, 2010.

[6] J.L. Caruso, Operations in equivariant $\mathbf{Z} / p$-cohomology, Math. Proc. Cambridge Philos. Soc. 126, 3 (1999), 521-541.

[7] D. Dugger, An Atiyah-Hirzebruch spectral sequence for $K R$-theory, $K$-Theory 35 (2005), 213-256 (2006).

[8] J.P.C. Greenlees and J.P. May, Equivariant stable homotopy theory, 277-323.

[9] M.A. Hill, M.J. Hopkins, and D.C. Ravenel, On the non-existence of elements of Kervaire invariant one, pre-print (2014), http://arxiv.org/abs/0908.3724.

[10] P. Hu and I. Kriz, Real-oriented homotopy theory and an analogue of the Adams-Novikov spectral sequence, Topology 40, 2 (2001), 317-399.

[11] L.G. Lewis, Jr., Change of universe functors in equivariant stable homotopy theory, Fund. Math. 148, 2 (1995), 117-158.

[12] L.G. Lewis, Jr., J.P. May, M. Steinberger, and J.E. McClure, Equivariant stable homotopy theory, vol. 1213 of Lecture Notes in Mathematics, Springer-Verlag, Berlin, 1986, With contributions by J.E. McClure.

[13] H.R. Margolis, Spectra and the Steenrod algebra, vol. 29 of North-Holland Mathematical Library, North-Holland Publishing Co., Amsterdam, 1983, Modules over the Steenrod algebra and the stable homotopy category.

[14] J.W. Milnor and J.C. Moore, On the structure of Hopf algebras, Ann. of Math. (2) 81 (1965), 211-264.

[15] D.C. Ravenel, Complex cobordism and stable homotopy groups of spheres, vol. 121 of Pure and Applied Mathematics, Academic Press Inc., Orlando, FL, 1986.

Nicolas Ricka ricka@math.univ-paris13.fr

Institut de Recherche Mathématique Avancée, UMR 7501, 7 rue René-Descartes, 67084 Strasbourg, France 